Nota de investigación

\title{
Evaluación de dos procedimientos de medición de color en granos de maíces pigmentados
}

\author{
Yolanda Salinas-Moreno \\ José Luis Ramírez Díaz \\ Ivone Alemán de la Torre \\ Edgardo Bautista-Ramírez \\ Alejandro Ledesma Miramontes ${ }^{\S}$ \\ Campo Experimental Centro Altos de Jalisco-INIFAP. Carretera Tepatitlán-Lagos de Moreno km 8, Tepatitlán \\ de Morelos, Jalisco. México. CP. 47600. (salinas.yolanda@inifap.gob.mx; ramirez.joseluis@inifap.gob.mx; \\ alemán.ivone@inifap.gob.mx; bautista.edgardo@inifap.gob.mx). \\ ${ }^{\S}$ Autor para correspondencia: ledesma.alejandro@inifap.gob.mx.
}

\section{Resumen}

El grano de maíz con pigmentos tipo antociano, no presenta una coloración homogénea en su superficie, por lo que la forma de presentar el grano para tomar la lectura de color afecta los resultados. En este trabajo se compararon dos formas de tomar la lectura de color en muestras de grano de colores diversos. Se emplearon muestras de grano de color azul morado (AZM), rojo cereza ( $\mathrm{RC}$ ), rojo ladrillo (RL), amarillo (AM) y blanco (BCO), este último como testigo. Los dos procedimientos para medir la coloración del grano fueron: montado sobre una base de plastilina, simulando como se encuentra en la mazorca (GMP) y grano en charola $(\mathrm{GCH})$. El color se determinó con un colorímetro Hunter-Lab Mini Scan. En los colores de grano AZM, RC y RL el procedimiento para tomar la lectura de color afectó las variables de color. En los maíces AZM, las diferencias se presentaron tanto en la luminosidad, como en hue y croma, en los RC y RL fue la variable hue la más afectada. En los maíces de grano AM y BCO, los valores de luminosidad, hue y croma fueron numéricamente cercanos entre las dos formas de tomar la lectura de color en el grano. De acuerdo con los resultados, en los maíces AZM, RC y RL el color debería tomarse en el GMP, para obtener valores en las variables de color, que se relacionan con la apreciación visual de color, en tanto que en los maíces de grano AM y BCO el procedimiento para tomar la lectura, si bien afecta las variables de color, el valor de hue corresponde con la apreciación visual del color.

Palabras clave: colorímetro, hue y croma, lectura de color, luminosidad.

Recibido: septiembre de 2021

Aceptado: octubre de 2021 
El color es un atributo físico de alimentos o productos, que puede detectarse de manera visual, pues el ojo humano es capaz de diferenciar millones de colores. Sin embargo, esta valoración carece de objetividad y puede variar según la percepción del individuo. Por tanto, el uso de instrumentos aptos para medir el color en función de variables objetivas es elemental para establecer caracterizaciones y comparaciones entre productos.

La determinación objetiva del color de cualquier producto sólido o liquido se realiza mediante un colorímetro, con el cual se obtiene las variables: luminosidad $\left(\mathrm{L}^{*}, 0 \%=\right.$ blanco, $100 \%=$ negro), a (verde en valores negativos y rojo en valores positivos), y $\mathrm{b}^{*}$ (amarillo en valores positivos y azul en valores negativos). A partir de $\mathrm{a}^{*} \mathrm{y} \mathrm{b}^{*}$ se calcula el ángulo de tono o hue $\left(\mathrm{h}^{\circ}\right)$ y el índice de saturación o croma $\left(C^{*}\right)$ (McGuire, 1992). La apreciación visual de color está altamente relacionada con el ángulo de tono determinado objetivamente (Jha, 2010; Owens et al., 2019). La manera en que se presenta la muestra para realizar la lectura influye en los valores de las variables de color. En productos sólidos, sean translúcidos u opacos, es importante la uniformidad del color en su superficie. Si esta es irregular, la zona en que se tome la lectura tendrá efecto en el dato de color obtenido (Wrolstad y Smith, 2010).

El grano de maíz se integra por las macroestructuras de pericarpio, endospermo, germen y pedicelo. El pericarpio envuelve al endospermo y al germen. En los granos de maíces pigmentados con presencia de polifenoles en la capa de aleurona, es común que el germen carezca de pigmento (Salinas et al., 1999), por lo que esta zona se aprecia de un tono distinto al resto del grano.

Esta situación provoca que cuando la medición del color se realiza sobre la superficie de los granos, los valores de ángulo de tono en maíces de grano rojo o azul morado sean similares a los que se asocian con un tono amarillo o amarillo verdoso (Espinosa-Trujillo et al., 2006).

Actualmente existe un interés renovado por el uso y aprovechamiento de los maíces nativos, los cuales presentan amplia diversidad en color, que es necesario caracterizar de manera objetiva y reproducible, por lo que el objetivo del trabajo fue evaluar dos procedimientos (grano en charola y grano sobre base de plastilinas) para tomar la lectura de color en el grano de maíces con diferentes colores de grano, y con base en los resultados, definir la forma más adecuada para que los valores de hue obtenidos corresponden con la apreciación visual del color en grano de maíz pigmentado.

Se utilizaron 20 accesiones de maíz con grano azul/morado (AZM) de cinco razas de maíz en las que es común este color de grano, 14 accesiones de maíz con grano rojo cereza (RC) de cuatro razas en las este color es común, siete accesiones de grano rojo ladrillo (RL) de cinco razas que presentan estas coloraciones, siete maíces mejorados de grano amarillo (AM) (comerciales y experimentales), y siete maíces de grano blanco (tres híbridos comerciales y cuatro accesiones de las razas Cónico y Chalqueño). Las accesiones fueron recolectadas de diferentes orígenes, pero el dato de color se obtuvo en todos los casos en muestras de cosecha reciente; el material mejorado provino del Programa de Maíz del Campo Experimental Centro Altos de Jalisco, del INIFAP.

Las muestras de maíz blanco se incorporaron al estudio como testigos, al ser el color más predominante y de mayor uniformidad de color en su superficie. El contenido de humedad de las muestras al momento de la medición se ubicó entre 11 y $12.5 \%$. Para la determinación del color, se utilizó un colorímetro Hunter Lab MiniScan, en escala CIELab, con iluminante 65 y un ángulo 
de observación de $10^{\circ}$. Para la lectura del color del grano, la muestra se presentó en dos formas: A) muestra de granos montada sobre una base de plastilina gris (GMP), simulando la forma en que el grano se encuentra en la mazorca (Figura 1A), B) muestra de granos (30 g) contenidos en una charola de aluminio (GCH) (Figura 1B).
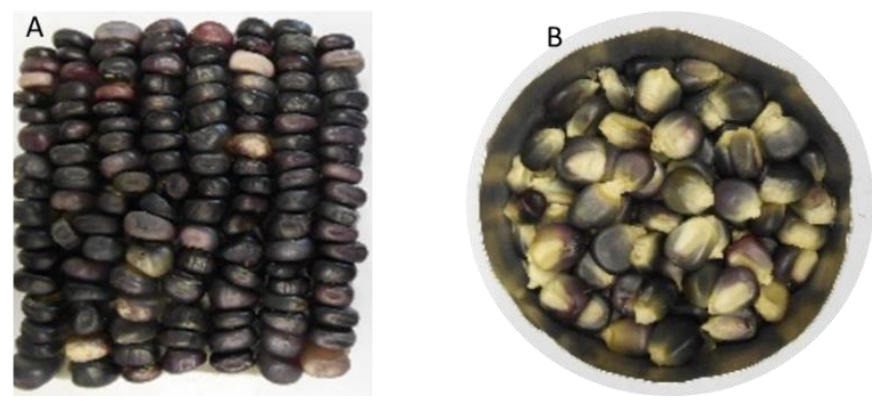

Figura 1. Forma en que se presentó la muestra de grano de maíz para tomar la lectura de color. A) grano montado sobre base de plastilina; y B) grano en charola.

En cada caso se realizaron seis lecturas (este número se definió para tener un error estándar de la media $\leq 5 \%$ ) con el equipo, en diferentes puntos de la superficie de la muestra. Se obtuvieron los valores de luminosidad ( $\left.\mathrm{L}^{*}\right), \mathrm{a}^{*} \mathrm{y} \mathrm{b}^{*}$. A partir de los valores de $\mathrm{a}^{*} \mathrm{y} \mathrm{b}^{*}$, se calcularon las variables de ángulo de tono $\left(\mathrm{h}^{\circ}\right)$, e índice de saturación de color (Croma) (McGuire, 1992). Los datos para cada color de grano se analizaron mediante comparación de medias entre los dos procedimientos para tomar la lectura de color, se consideró el número de muestras analizadas para cada color de grano, como repeticiones.

En los maíces de grano AZM, los valores de luminosidad $\left(\mathrm{L}^{*}\right)$ fueron menores y de mayor variabilidad en el GMP, con relación al GCH, lo que significa que el colorímetro detectó como más claro el GCH que el GMP. El valor promedio de esta variable fue menor cuando el color se tomó en el GMP en relación con el GCH (Cuadro 1). En la variable a*, el GMP reportó valores desde 0.63 hasta 6.28, en tanto que en el GCH mostró sólo valores positivos (0.41 a 3.62). Esto significa que, en el GMP, el colorímetro detectó tonalidades desde verde (-0.63) hasta rojo (6.28), en tanto que en el GCH solo detectó tonalidades rojas al presentar valores positivos (Jha, 2010).

Cuadro 1. Valores de las variables de color del grano de maíz obtenidos con dos procedimientos para tomar la lectura.

\begin{tabular}{|c|c|c|c|c|c|c|c|}
\hline \multirow{3}{*}{ Color de grano } & \multirow{3}{*}{$\begin{array}{l}\text { Criterio } \\
\text { estadístico }\end{array}$} & \multicolumn{6}{|c|}{ Variables de color } \\
\hline & & \multicolumn{2}{|c|}{$\mathrm{L}^{*}(\%)$} & \multicolumn{2}{|c|}{$a^{*}$} & \multicolumn{2}{|c|}{$b^{*}$} \\
\hline & & GMP & $\mathrm{GCH}$ & GMP & $\mathrm{GCH}$ & GMP & $\mathrm{GCH}$ \\
\hline \multirow{5}{*}{$\begin{array}{l}\text { Azul morado }(\mathrm{n}= \\
20)\end{array}$} & Valor mínimo & 16.91 & 39.32 & -0.63 & 0.41 & -1.02 & 5.96 \\
\hline & Valor máximo & 31.21 & 49.39 & 6.28 & 3.62 & 2.78 & 13.91 \\
\hline & Media & $25.18 \mathrm{~b}$ & $44.39 \mathrm{a}$ & $2.81 \mathrm{a}$ & $1.72 \mathrm{~b}$ & $0.73 b$ & $10.19 \mathrm{a}$ \\
\hline & $\mathrm{CV}(\%)$ & 14.8 & 71.4 & 149.8 & 6.5 & 50.9 & 22.9 \\
\hline & DSH & \multicolumn{2}{|c|}{2.13} & \multicolumn{2}{|c|}{1.01} & \multicolumn{2}{|c|}{1.17} \\
\hline
\end{tabular}




\begin{tabular}{|c|c|c|c|c|c|c|c|}
\hline \multirow{3}{*}{ Color de grano } & \multirow{3}{*}{$\begin{array}{l}\text { Criterio } \\
\text { estadístico }\end{array}$} & \multicolumn{6}{|c|}{ Variables de color } \\
\hline & & \multicolumn{2}{|c|}{$\mathrm{L}^{*}(\%)$} & \multicolumn{2}{|c|}{$\mathrm{a}^{*}$} & \multicolumn{2}{|c|}{$b^{*}$} \\
\hline & & GMP & $\mathrm{GCH}$ & GMP & $\mathrm{GCH}$ & GMP & $\mathrm{GCH}$ \\
\hline \multirow[t]{5}{*}{ Rojo cereza $(n=14)$} & Valor mínimo & 26.59 & 31.42 & 11.57 & 7 & 5.32 & 9.3 \\
\hline & Valor máximo & 46.52 & 55.94 & 20.03 & 17.32 & 13.37 & 16.18 \\
\hline & Media & $34.41 \mathrm{~b}$ & $42.66 \mathrm{a}$ & $17.12 \mathrm{a}$ & $11.26 \mathrm{~b}$ & $9.14 \mathrm{~b}$ & $12.97 \mathrm{a}$ \\
\hline & $\mathrm{CV}(\%)$ & 19.9 & 14.3 & 27 & 15.8 & 29.6 & 17.1 \\
\hline & DSH & \multicolumn{2}{|c|}{5.27} & \multicolumn{2}{|c|}{2.27} & \multicolumn{2}{|c|}{1.82} \\
\hline \multirow[t]{5}{*}{ Rojo ladrillo $(\mathrm{n}=7)$} & Valor mínimo & 18.5 & 27.34 & 14.24 & 13.14 & 7.04 & 11.69 \\
\hline & Valor máximo & 26.61 & 40.71 & 23.01 & 23.43 & 18.93 & 22.15 \\
\hline & Media & $22.93 \mathrm{~b}$ & $35.23 \mathrm{a}$ & $19.05 \mathrm{a}$ & $16.98 \mathrm{a}$ & $11.87 \mathrm{~b}$ & $17.5 \mathrm{a}$ \\
\hline & $\mathrm{CV}(\%)$ & 18.2 & 17.6 & 33.3 & 13.7 & 21.1 & 20.8 \\
\hline & DSH & \multicolumn{2}{|c|}{5.24} & \multicolumn{2}{|c|}{4.04} & \multicolumn{2}{|c|}{4.42} \\
\hline \multirow[t]{5}{*}{ Amarillo $(n=7)$} & Valor mínimo & 57.3 & 60.24 & 7.65 & 11.02 & 41.04 & 36.2 \\
\hline & Valor máximo & 70.81 & 63.5 & 19.6 & 16.4 & 55.7 & 46.6 \\
\hline & Media & $63.3 \mathrm{a}$ & $61.94 \mathrm{a}$ & $13.78 \mathrm{a}$ & $13.33 \mathrm{a}$ & $49.92 \mathrm{a}$ & $40.71 \mathrm{~b}$ \\
\hline & $\mathrm{CV}(\%)$ & 8.4 & 39.6 & 10.3 & 2.2 & 12.6 & 10.3 \\
\hline & DSH & \multicolumn{2}{|c|}{4.52} & \multicolumn{2}{|c|}{4.71} & \multicolumn{2}{|c|}{5.46} \\
\hline \multirow{5}{*}{$\begin{array}{c}\text { Blanco }(n=4) \\
\text { Nativos }\end{array}$} & Valor mínimo & 60.1 & 57.22 & 3.51 & 3.59 & 19.55 & 18.87 \\
\hline & Valor máximo & 65.92 & 62.14 & 11.01 & 9.47 & 45.55 & 22.35 \\
\hline & Media & $63.02 \mathrm{a}$ & $60.4 \mathrm{a}$ & $6.64 \mathrm{a}$ & $5.52 \mathrm{a}$ & $33.05 \mathrm{a}$ & $20.27 \mathrm{a}$ \\
\hline & $\mathrm{CV}(\%)$ & 4.4 & 67.5 & 51.5 & 3.7 & 75.2 & 8.5 \\
\hline & $\mathrm{DSH}$ & \multicolumn{2}{|c|}{4.34} & \multicolumn{2}{|c|}{5.4} & \multicolumn{2}{|c|}{14.2} \\
\hline \multirow{5}{*}{$\begin{array}{c}\text { Blanco }(n=3) \\
\text { Mejorados }\end{array}$} & Valor mínimo & 73.9 & 68.6 & 1.2 & 2.7 & 20.7 & 24.4 \\
\hline & Valor máximo & 74.7 & 70.7 & 1.8 & 2.8 & 22.3 & 25.9 \\
\hline & Media & 74.38 a & $69.65 \mathrm{~b}$ & $1.46 \mathrm{~b}$ & $2.75 \mathrm{a}$ & $21.59 \mathrm{~b}$ & $25.24 \mathrm{a}$ \\
\hline & $\mathrm{CV}(\%)$ & 0.6 & 18.6 & 3.6 & 1.5 & 3.1 & 3 \\
\hline & DSH & \multicolumn{2}{|c|}{1.86} & \multicolumn{2}{|c|}{0.46} & \multicolumn{2}{|c|}{1.73} \\
\hline
\end{tabular}

Medias con letras iguales en hileras, para cada parámetro de color, no son estadísticamente diferentes (DMS, 0.05). $\mathrm{DSH}=$ diferencia significativa honesta $(p \leq 0.05)$.

Con la variable $b^{*}$, en el GMP la variabilidad fue desde -1.02 (tonalidad azul) hasta ligeramente amarillo (2.78), en tanto que para el GCH la variabilidad fue mayor (5.96 a 13.91), pero en la tonalidad amarilla. Se presentaron diferencias estadísticas para $a^{*}$ y b* entre el GMP y GCH. En los maíces de grano RC ocurrió algo similar a lo observado en los maíces de grano AZM. De acuerdo con las variables de color, el grano es más oscuro (menor valor de $\mathrm{L}^{*}$ ) y rojo (menor valor de $^{*}$ ) (Wrolstad y Smith, 2010) cuando se toma la lectura en el GMP, que cuando se toma en el $\mathrm{GCH}$. En las tres variables de color se presentó diferencia estadística $(p<0.05)$ entre las dos formas de presentar el grano para tomar la lectura de color. 
En los maíces de grano RL, la L* y el valor de $b^{*}$ fueron menores $(p<0.05)$ en el GMP con relación al $\mathrm{GCH}$, por lo que se apreciaron como más oscuros. En los maíces de grano AM, los valores promedio de las variables $\mathrm{L}^{*}$ y a* fueron iguales $(p<0.05)$ entre GMP y GCH. La variable b* resultó mayor en el GMP con relación al GCH, lo que significa mayor tonalidad amarilla en los primeros.

Los maíces nativos de grano BCO presentaron valores iguales de las variables de color entre el GMP y GCH, por lo que no se puede tomar la lectura en GCH y evitar el trabajo de ponerlo sobre plastilina. En los maíces BCO mejorados, que son de tipo dentado, la fracción harinosa del endospermo se concentra en la corona del grano, por lo que al tomar la lectura de color en el GMP los valores de $\mathrm{L}^{*}$ resultan mayores que cuando se toma en el GCH. En $\mathrm{a}^{*} \mathrm{y} \mathrm{b}^{*}$, por el contrario, los valores fueron más elevados en el GCH, con relación al GMP.

En la Figura 2 se presentan los resultados de hue-croma, que representan la cromaticidad (Jha, 2010), de las muestras analizadas para cada color de grano, con los dos procedimientos en que se tomó la lectura. En los maíces AZM se observó una clara diferencia de los valores de hue, entre los dos procedimientos para tomar el color (Figura 2A). En el GMP los valores se relacionan con tonos azul morado y rojo morado, mientras que, en el $\mathrm{GCH}$, se asocian con tonos amarillos.
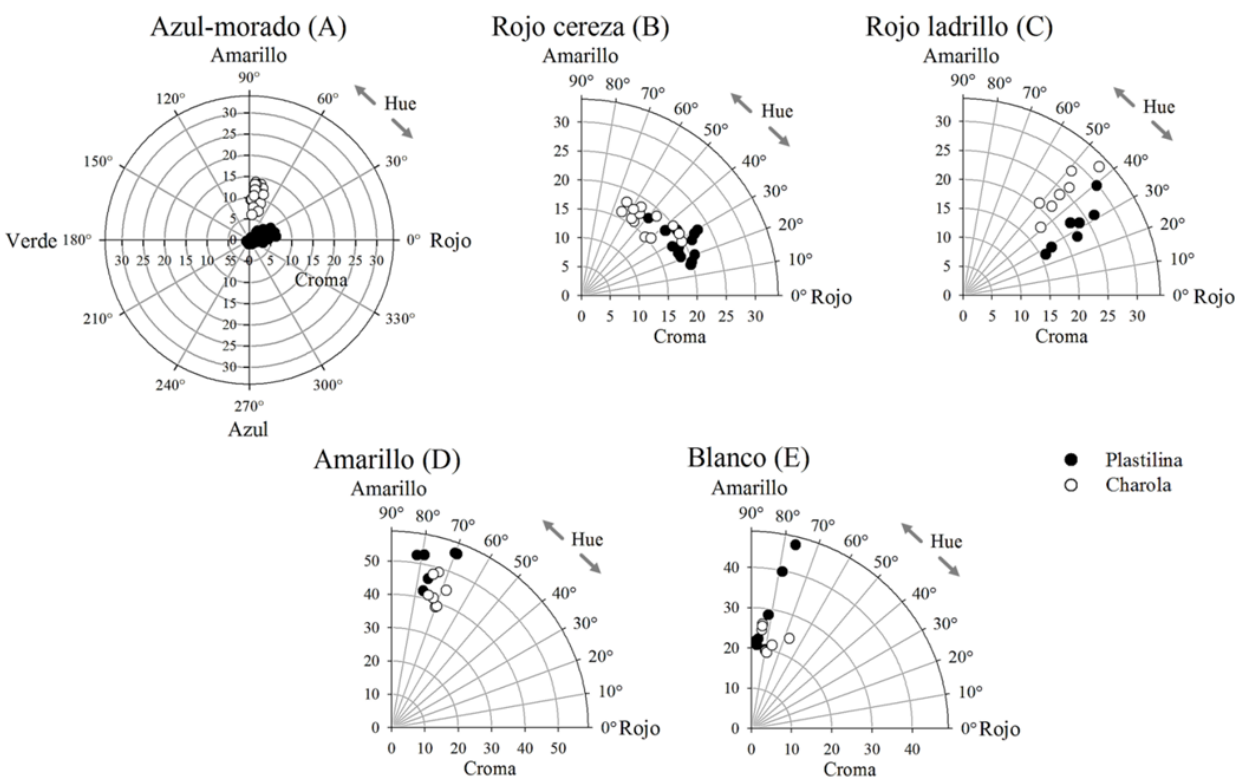

Charola

Figura 2. Distribución en el plano hue vs croma de las muestras de grano de maíz de los colores analizados.

Los valores de croma en el GMP fueron menores a 7, en tanto que en el GCH variaron entre 6 y 14. Esto significa mayor presencia de tonos grises en el GMP con relación al GCH. De las variables de cromaticidad, el ángulo de tono o hue es la más asociada con la percepción visual de color (Jha, 2010; Wrolstand y Smith, 2010) por lo que los resultados en esta variable pueden servir de guía para saber si el color se determina de la manera correcta. Espinosa-Trujillo et al. (2006) informaron valores promedio de hue y croma de $108.5 \pm 9.7^{\circ}$ y $5.8 \pm 1.5$, en ese orden, en 18 poblaciones de maíz con grano azul. Estos valores corresponden a un tono amarillo verdoso, con bajo índice de saturación de color. 
Los autores midieron el color sobre una muestra de granos contenidos en una charola. En maíces de grano morado, con el pigmento concentrado en el pericarpio, los valores de hue en 20 líneas variaron entre 10.2 y $73.5^{\circ}$ que según los autores no correspondieron con el color visual observado, que fue morado intenso a púrpura (Mendoza-Mendoza et al., 2017). En este último estudio no se menciona la forma que el grano se presentó para medir la lectura con el colorímetro.

En los maíces de grano RC las diferencias en el procedimiento de tomar la lectura se manifestaron en los valores de hue. Cuando se tomó en el GMP, hue se asoció con una tonalidad roja, en tanto que cuando se tomó en $\mathrm{GCH}$, lo hizo con un tono anaranjado amarillo (Figura 2B). En los valores de croma, los datos obtenidos entre las dos formas de tomar la lectura fueron cercanos a 15 y 20.

En los maíces de grano RL los valores de hue, fueron ligeramente bajos, y por tanto asociados con tonos más rojos para el GMP que el GCH, cuyos valores de hue corresponden a tono anaranjado (Figura 2C). Los valores de hue y croma reportados por Espinosa-Trujillo et al. (2006) para maíces de grano rojo fueron de $75.7 \pm 7.1^{\circ}$ y $5.7 \pm 1.1^{\circ}$, respectivamente. Estos son similares a los obtenidos en maíces $\mathrm{RC}$, con el procedimiento de $\mathrm{GCH}$ y se asocian con tonalidades amarillas-anaranjadas.

En los maíces de grano AM no se observó una diferencia en las variables de cromaticidad, entre los dos procedimientos de medir el color del grano (GMP y GCH). Los valores de hue variaron entre 69.3 y $81.6^{\circ}$ en el GMP, y entre 68.3 y $74.8^{\circ}$, en el GCH, que son parecidos al valor promedio de $70 \pm 0.75^{\circ}$ reportado por Vázquez-Carrillo et al. (2011) en seis híbridos con esta coloración de grano. En los maíces de grano BCO, en donde se incluyeron tanto nativos como híbridos, el valor de hue fue similar entre las dos formas de tomar la lectura. Las muestras que se dispararon en sus valores de croma, en el GMP, corresponden a los maíces nativos, que presentaron entre ellos amplias variaciones en los valores de $\mathrm{a}^{*} \mathrm{y} \mathrm{b}^{*}$, y que son más bien de color cremoso (Cuadro 1).

\section{Conclusiones}

En los colores de grano azul morado, rojo cereza y rojo ladrillo el procedimiento de toma de lectura de color en el grano afectó los parámetros de color. Los valores de hue obtenidos cuando la lectura se tomó en el grano sobre base de plastilina correspondieron con el color visual del grano, por lo que en estos maíces se recomienda tomar la lectura de color sobre la superficie de granos montados en plastilina, cuando no se tenga la mazorca. En los maíces de grano amarillo y blanco el hue fue parecido entre los dos procedimientos de toma de color, por lo que se puede realizar con cualquiera de los dos procedimientos.

\section{Literatura citada}

Espinosa-Trujillo, E.; Mendoza-Castillo, M. C. y Castillo-González, F. 2006. Diversidad fenotípica entre poblaciones de maíz con diferentes grados de pigmentación. Rev. Fitotec. Mex. 29(2):19-23.

Jha, S. N. 2010. Colour measurements and modeling. In: nondestructive evaluation of food quality: theory and practice, Shyam N. Jha. Ed. Springer. 23-24 pp.

McGuire, R. G. 1992. Reporting of objective color measurements. HortScience. 27(12):1254-1255.

Mendoza-Mendoza, C. G.; Mendoza-Castillo, M. C.; Delgado-Alvarado, A.; Castillo-González, F.; Kato-Yamakake, T. A. y Cruz-Izquierdo, S. 2017. Antocianinas totales y parámetros de color en líneas de maíz morado. Rev. Fitotec. Mex. 40(4):471-479. 
Owens, B. F.; Mathew, D.; Diepenbrock, C. H.; Tiede, T.; Wu, D.; Mateos-Hernandez, M.; Gore M. A. and Rocheford, T. 2019. Genome-Wide association study and pathway-level analysis of kernel color in maize. G3 Genes Genomes Genetics. 9(6):1945-1955. https://doi.org/ 10.1534/g3.119.400040.

Salinas, M. Y.; Soto, H. M.; Martínez, B. F.; González, H. V. y Ortega, P. R. 1999. Análisis de antocianinas en maíces de grano azul y rojo provenientes de cuatro razas. Rev. Fitotec. Mex. 22(2):161-174.

Vázquez-Carrillo, G.; García-Lara, S.; Salinas-Moreno, Y.; Bergvinson, D. J. and Palacios-Rojas, N. 2011. Grain and tortilla quality in landraces and improved maize grown in the highlands of Mexico. Plant Foods Human Nutr. 66(2):203-208. https://doi.org/10.1007/s11130-0110231-7.

Wrolstad, E. R. and Smith, D. E. 2010. Color analysis. In: Food analysis. Nielsen, S. S. (Ed.). Fourth edition. Springer. 575-586 pp. http://dx.doi.org/10.1007/978-1-4419-1478-1. 